\title{
Pain Severity in Relation to the Final Menstrual Period in a Prospective Multiethnic Observational Cohort: Results from the Study of Women's Health Across the Nation (SWAN)
}

\author{
Yvonne C. Lee, MD, MMSc ${ }^{1}$, Arun S. Karlamangla, MD, $\mathrm{PhD}^{2}$, Zhi Yu, MS ${ }^{1}$, Chih-Chin Liu, \\ $\mathbf{P h D}^{1}$, Joel S. Finkelstein, MD ${ }^{3}$, Gail A. Greendale, $\mathbf{M D}^{2}$, Siobán D. Harlow, $\mathbf{P h D}^{4}$, and Daniel \\ H. Solomon, MD, MPH ${ }^{1}$ \\ ${ }^{1}$ Division of Rheumatology, Immunology and Allergy, Harvard Medical School, Brigham and \\ Women's Hospital, 75 Francis Street, PBB-B3, Boston, MA 02115, USA \\ 2Division of Geriatrics, David Geffen School of Medicine at UCLA, 10945 Le Conte Avenue, Suite \\ 2339, Los Angeles, CA 90095, USA \\ ${ }^{3}$ Endocrine Unit, Department of Medicine, Massachusetts General Hospital, 50 Blossom Street, \\ Boston, MA 02114, USA \\ ${ }^{4}$ Department of Epidemiology, University of Michigan, 1415 Washington Heights, Ann Arbor, MI \\ 48109, USA
}

\begin{abstract}
The development of pain is common in midlife, resulting in increased healthcare utilization and costs. The aim of this study was to determine the longitudinal trajectory of overall bodily pain among women during the transition between the reproductive years and menopause. We conducted analyses on a community-based, longitudinal cohort of women enrolled in the Study of Women's Health Across the Nation. One thousand four hundred and ninety-five women met inclusion criteria, including: 1) defined date of the final menstrual period (FMP) and 2) complete data on SF-36 bodily pain. The primary exposure was time to/from FMP. The primary outcome was the rate of change in SF-36 bodily pain, measured on a scale of 0-100 with 100 being the most severe pain. We performed within-person trajectory analyses using piecewise regression following nonparametric modeling of functional forms. Mean bodily pain score at the time of the FMP was 29. Mean bodily pain increased at a rate of 0.26 per year during the transmenopause (the interval spanning 4.5 years prior to the FMP through 0.5 years after FMP), and decreased at a rate of 0.23 per year after that. Depression and sleep problems were associated with greater increases in pain
\end{abstract}

Corresponding author and person to whom reprint requests should be addressed: Yvonne C. Lee, MD, MMSc, Division of Rheumatology, Immunology and Allergy, Brigham and Women's Hospital, 75 Francis Street, PBB-B3, Boston, MA 02115, Phone: 617-732-8736, Fax: 617-713-3030, ylee9@partners.org.

Publisher's Disclaimer: This is a PDF file of an unedited manuscript that has been accepted for publication. As a service to our customers we are providing this early version of the manuscript. The manuscript will undergo copyediting, typesetting, and review of the resulting proof before it is published in its final citable form. Please note that during the production process errors may be discovered which could affect the content, and all legal disclaimers that apply to the journal pertain.

Disclosures:

Conflicts of interest: YL, AK, CL, JF, GG, SH and DHS have nothing to declare. 
during the late reproductive years, whereas abdominal cramps at baseline predicted greater decreases in pain during the late reproductive years.

Perspectives-This article demonstrates that bodily pain increases during the transmenopause and then diminishes during postmenopause. These differences may reflect differences in underlying mechanisms of pain in the two periods. Although mean changes were small and unlikely to be clinically meaningful, the magnitude of change varied across subgroups of women.

\section{Keywords}

Pain; perimenopause; menopause; female; aging

\section{Introduction}

Chronic pain is common, affecting approximately 116 million people in the United States ${ }^{22}$. Most chronic pain patients are women, and women with chronic pain experience greater pain-related interference with function than men. ${ }^{21,38}$ In women, the prevalence of pain across the lifespan is not well characterized. Most studies have been cross-sectional or have grouped men and women together when calculating prevalence rates. A longitudinal study using data from the National Population Health Survey reported that the prevalence of chronic pain increased from $11.9 \%$ among women between $25-39$ years old to $28.4 \%$ among women over 70 years old. ${ }^{31}$ Studies focusing on pain among women before, during and after the menopause transition have yielded conflicting results, with some studies suggesting that the overall prevalence of pain peaks in the postmenopausal years ${ }^{26}$ and other studies showing no significant changes in pain among women transitioning from pre/perimenopause to postmenopause compared to women who remained premenopausal. ${ }^{24}$ The conflicting data are, in part, due to difficulties in studying pain during this period when hormonal fluctuations occur in conjunction with other age-related physiologic changes (e.g., increases in obesity, osteoarthritis, etc.). ${ }^{2,} 6,10,50$

The strongest studies demonstrating a link between sex hormone levels and pain have been clinical trials examining the effects of menopausal hormone therapy. Based on a post-hoc analysis of data from the Women's Health Initiative, Chlebowski et al. found that treatment with daily oral conjugated equine estrogens was associated with a decrease in the frequency and intensity of joint pain. ${ }^{4}$ However, Stening et al. noted no significant difference in selfreported pain, pain threshold or pain tolerance among women with fibromyalgia who received transdermal $17 \beta$-estradiol vs. those who received placebo. ${ }^{37}$ Differences in the outcomes of these studies may result from differences in the study population and outcome measures.

Data from large observational cohort studies are needed to better understand the natural history of changes in pain as women age, in the absence of exogenous menopausal hormone therapy (HT). Most previous studies have used bleeding patterns to categorize women as premenopausal, perimenopausal or postmenopausal. ${ }^{7,25,39}$ These reproductive aging categories, however, encompass large periods of time, which are not equivalent. Hormonal levels may differ substantially within each category, depending on the exact timing from the 
final menstrual period (FMP). ${ }^{9}, 34$ Hormone levels may also vary considerably between women and depend on the timing of the measurements during each menstrual cycle.

The objective of this study was to determine the longitudinal trajectory of bodily pain in relation to the time before/after the FMP among women not taking exogenous hormones, followed from the late reproductive years to postmenopause. We planned this study to examine changes in overall bodily pain, rather than specific types of pain, because overall bodily pain significantly impacts quality of life, independent of etiology. We hypothesized that levels of bodily pain vary by time before and after the FMP, and, specifically, that bodily pain increases during transmenopause (as women approach and pass the FMP) and continues to increase in the postmenopause.

\section{Methods}

\section{Study design and population}

The study population was derived from the Study of Women's Health Across the Nation (SWAN), a multi-site, community-based, prospective observational cohort of 3,297 women. The primary aims of SWAN are to: 1) characterize the natural history of reproductive aging through the late postmenopause; 2) evaluate the impact of reproductive aging on health outcomes clinically relevant to women in their $60 \mathrm{~s}$ and $70 \mathrm{~s}$; and 3 ) identify potential underlying mechanisms linking reproductive aging and health.

To be included in SWAN, women had to be between 42 and 52 years old in 1996-1997 and report at least 1 menstrual period within 3 months of the baseline study visit. Other inclusion criteria included: 1) intact uterus and at least 1 intact ovary and 2) no hormonal medications within 3 months of the baseline study visit. Each site recruited white women and one nonwhite ethnic/racial group (Hispanic in Newark; African American in Pittsburgh, Boston, Detroit and Chicago; Chinese in Oakland; and Japanese in Los Angeles). Follow-up visits occur approximately every year. Data up to and including follow-up visit 12 were available for this analysis. Written informed consent was obtained from all women. Study protocols and forms were approved by all SWAN site Institutional Review Boards.

The sample size of 1,495 women was determined by the number of women with available data on: 1) date of the FMP or sufficient information to estimate the date of the FMP and 2) data on SF-36 bodily pain measures. Eighty women who reported surgically-induced menopause were excluded. Two women taking tamoxifen, two women taking a $\mathrm{GnRH}$ agonist and one woman taking an opioid pain medication were excluded at baseline. Women who subsequently started taking opioid pain medications, HT, tamoxifen and/or GnRH agonists were censored at the time of first reported use.

\section{Measures}

Data were included from baseline (1996-1997) to visit 12 (2009-2011). Study visits occur each year and include physical measures (e.g., weight, height), interviewer-administered and self-administered questionnaires (translated into Cantonese, Japanese and Spanish). 
The primary outcome was the rate of change in the SF- 36 bodily pain index. ${ }^{44}$ This index includes 2 questions that assess pain magnitude and interference: 1) How much bodily pain have you had during the past 4 weeks, and 2) During the past 4 weeks, how much did pain interfere with your normal work (including both work outside the home and housework)? Traditionally, the SF-36 pain bodily index is scored such that high numbers reflect low pain. For ease of interpretation, we reversed the SF-36 pain index such that $0=$ no pain and $100=$ severe pain. Secondary outcomes were the individual SF-36 pain magnitude and pain interference items. For ease of comparison with the primary analysis, these measures were each converted to a $0-100$ scale using the conversion tables in the SF- 36 manual ${ }^{45}$ and reversed such that $0=$ no pain or pain interference and $100=$ severe pain or pain interference.

The primary exposure was time prior to or after the FMP, calculated as the number of years between the SF-36 bodily pain measurement and the FMP date. The FMP was defined as the menstrual period immediately before the first visit when the participant was classified as postmenopausal (12 months of amenorrhea). When the exact date of FMP was not available because the last bleeding date was missing or because the participant missed 1-2 visits before the first postmenopausal visit, the date of FMP was estimated as described in previous manuscripts. ${ }^{12,13}$

Age at FMP, self-reported ethnicity/race, socioeconomic status (annual income $<20 \mathrm{~K}$, 20-34K, 35-49K, 50-74K, $275 \mathrm{~K}$ ) and smoking status (yes/no) were obtained from the annual SWAN interviews. BMI was calculated from heights and weights measured annually. Depression (yes/no) was defined as a Center for Epidemiologic Studies Depression Scale (CES-D) score $\geq 16 .{ }^{5,47}$ Sleep problems were assessed with 4 questions about sleep quality, difficulty falling asleep, waking up at night and waking up earlier than planned (yes/no). We also included a covariate based on the question, "During the last year, have you had any of the following during at least half of your menstrual periods or in the week before them? A. Abdominal pain or cramps?"

\section{Statistical analysis}

The primary outcome was the annual rate of change in SF-36 bodily pain. To analyze change in bodily pain in relation to age and FMP date, we performed a within person trajectory analysis, using a staged approach. ${ }^{14}$ This modeling method takes advantage of prospectively collected data regarding the FMP to describe the menopause transition, rather than depending on descriptions of menstrual bleeding, which yield broad categorizations encompassing large, heterogeneous periods of time.

Stage 1 consisted of nonparametric, loess-based selection of the functional form of the trajectory of SF-36 bodily pain in relationship to the date of the FMP. To fit the model, observed data were restricted to the time range from 10 years before the FMP to 10 years after the FMP. Observations at the extremes of the distribution of time from FMP $(<2.5 \%$ and $>97.5 \%$ ) were trimmed to prevent undue influence on the trajectories by a small number of extreme values, resulting in a time range from 8.8 years before the FMP to 8.4 years after the FMP. These analyses suggested a piecewise linear trajectory with two inflexion points (or knots), one at 4.5 years before the FMP and a second at 0.5 years after the FMP. 
Stage 2 used mixed effects, linear regression models to identify the placement of knots for bodily pain trajectory. We examined the change in the explained proportion of withinwoman variance when the knots were varied around FMP minus 4.5 years and FMP plus 0.5 years. The identified knots divided the follow-up period into segments which we named: 1) late reproductive: 8.8 to 4.5 years before the FMP, 2) transmenopause: 4.5 years before the FMP to 0.5 years after the FMP, 3) postmenopause: 0.5 to 8.4 years after the FMP.

Stage 3 comprised of mixed effects, piecewise linear regression with fixed knots (using mixed effects modeling) to estimate the annual rate of change (slope) of bodily pain scores during each of the linear segments of the trajectory. Mixed effects models use data from all women, even if they have missing data for some visits. Unadjusted analyses were used to determine the mean trajectory in the population. The effects of time-fixed predictors (study site, demographic factors, abdominal cramps at baseline) and time-varying covariates (smoking, BMI, depression and sleep problems) were examined in multivariable adjusted analyses. Because women with earlier onset of the FMP are, by definition, younger at the time of FMP and less likely to have conditions that commonly cause pain in the elderly (e.g., joint damage from osteoarthritis), we included age at FMP as a predictor in the multivariable analyses. Abdominal cramps at baseline (when women were still menstruating) was included to allow for different trajectories of pain over the menopause transition in women who report abdominal pain due to menstrual periods. Baseline variables were added to the mixed-effects piecewise models as fixed effects on the intercept (level of pain at the FMP) and slopes (rate of change in pain). Clinical covariates (smoking, BMI, depression and sleep problems) were updated over time because these variables have been shown to impact self-reported pain in women, ${ }^{20,35,42,49}$ and we were interested in examining the rates of change in pain, independent of changes in these variables. Only baseline values of these time-varying variables were allowed to affect rates of change in pain (slopes). Current values were allowed only to affect current levels of pain (intercepts).

Cumulative change in SF-36 bodily pain was calculated from 7 years before the FMP to 5 years after the FMP. We chose to focus on this 12-year period rather than reporting the cumulative change over a longer period of time (e.g., the 17.2 years surrounding the FMP from which data were obtained) to minimize the likelihood of small estimations errors in slope causing large errors in the estimation of cumulative change.

To understand the meaning of the changes in SF-36 bodily pain, we performed secondary analyses, separately examining changes in each component of the SF-36 bodily pain measure (pain magnitude and pain interference). In the first step, we created plots of pain magnitude and pain interference over time from FMP, using local regression to fit a smooth curve to the data points. Because we observed similar patterns of change between pain magnitude and the overall SF-36 bodily pain measure, we applied the same inflexion points determined from the primary SF-36 bodily pain analysis to the pain magnitude analysis. Because different patterns of change were noted for pain interference compared to pain magnitude and the overall bodily pain measure, different knots (at 0.5 years and 5.5 years after the FMP) were chosen for the pain interference analysis. These knots divided the follow-up period into different segments, compared to the bodily pain and pain magnitude analyses: 1) late reproductive and transmenopause: 8.8 years before the FMP to 0.5 years 
after the FMP; 2) early postmenopause: 0.5 to 5.5 years after the FMP, and 3) late postmenopause: 5.5 to 8.4 years after the FMP. Rates of change were calculated in the same manner as described for Stage 3 of the SF-36 bodily pain analysis. All statistical analyses were performed using the SAS 9.2 software package (SAS Institute, Cary, NC, USA).

\section{Results}

\section{Participant characteristics}

Among the 3,297 women in SWAN, 1,714 were excluded due to missing data on the FMP (Figure 1). Eighty-five women were excluded because they had surgically-induced menopause or were taking medications that affected hormone function or pain, and three women were excluded because they only provided data at the extremes of the distribution (< $2.5 \%$ and $>97.5 \%$ ) of time from FMP. The final study sample consisted of 1,495 women. Six hundred and forty-three (43.2\%) were Caucasian; 449 (30.1\%) were African American; 155 (10.4\%) were Japanese; 145 (9.7\%) were Chinese, and 98 (6.6\%) were Hispanic (Table 1). The mean age at baseline was $46.8 \pm 2.5$ years. The mean age at the FMP was $51.9 \pm 2.7$ years. At baseline, $856(57.7 \%)$ women reported abdominal pain associated with menstruation. The mean SF-36 bodily pain score at baseline was $28.7 \pm 22.1$, consistent with a low level of pain. The mean number of observations per woman over the entire 17.2-year study period was $6.8 \pm 1.9$. On average, $4.1 \pm 1.7$ observations occurred before the FMP and $3.0 \pm 1.4$ observations occurred after the FMP.

\section{Mean trajectories of pain scores}

Overall bodily pain-The inflexion points that explained the largest proportion of the within-woman variance were 4.5 years before the FMP and 0.5 years after the FMP. The mean pain level was 29.1 (out of 100) at the time of FMP. During the late reproductive years ( 8.8 to 4.5 years before the FMP), pain levels remained essentially constant, with a slope (change per year) of $0.01(95 \% \mathrm{CI}=-0.45,0.47)$ - statistically not different from zero slope (Figure 2, Table 2). In contrast, pain increased on average by 0.26 per year $(95 \% \mathrm{CI}=0.01$, 0.51 ) during transmenopause (4.5 years before the FMP to 0.5 years after the FMP) and decreased on average by 0.23 per year $(95 \% \mathrm{CI}=-0.44,-0.03)$ in postmenopause $(0.5-8.4$ years after the FMP). The rate of change in bodily pain during transmenopause was significantly different from the rate of change in bodily pain during postmenopause ( $P=$ 0.01 ). The mean cumulative change over the 12 -year period from 7 years before the FMP to 5 years after the FMP was not statistically different from zero (Table 2).

Pain magnitude-The best inflexion points for the trajectory of pain magnitude were the same as those for overall bodily pain, namely 4.5 years before the FMP and 0.5 years after the FMP. During the late reproductive years (before 4.5 years before the FMP), pain magnitude was essentially stable. The mean slope was not statistically different from zero (Supplementary Figure 1 and Supplementary Table 1). Pain magnitude increased on average by 0.41 per year during transmenopause ( 4.5 years before the FMP to 0.5 years after the FMP; $95 \% \mathrm{CI}=0.13,0.69)$ and subsequently decreased on average by 0.29 per year in postmenopause ( $95 \% \mathrm{CI}=-0.52,-0.06$ ). The rate of change in pain magnitude during transmenopause was significantly different from the rate of change in pain magnitude during 
the postmenopause $(P=0.002)$. The mean cumulative change over the 12 -year period from 7 years before the FMP to 5 years after the FMP was statistically not different from zero.

Pain interference-Because different patterns of change were noted for pain interference compared to bodily pain, different inflexion points were chosen for the pain interference analysis. These inflexion points divided the follow-up period into different segments: 1) late reproductive and transmenopause: 8.8 years before the FMP to 0.5 years after the FMP; 2) early postmenopause: 0.5 to 5.5 years after the FMP, and 3) late postmenopause: 5.5 to 8.4 years after the FMP. During the late reproductive years and transmenopause, pain interference was essentially constant. The rate of change was statistically not different from zero (Supplementary Figure 2 and Supplementary Table 2). Pain interference decreased on average during early postmenopause by 0.41 per year $(95 \% \mathrm{CI}=-0.69,-0.13)$ and subsequently increased by 1.08 per year in late postmenopause $(95 \% \mathrm{CI}=0.08,2.07)$. The rate of change in pain interference during early postmenopause was significantly different from the rates of change in pain interference during the late reproductive stage/ transmenopause $(P=0.009)$ and late postmenopause $(P=0.01)$. The mean cumulative change over the 12-year period from 7 years before the FMP to 5 years after the FMP was statistically not different from zero.

\section{Changes in overall bodily pain according to individual clinical variables}

Age at FMP had no associations with either level of pain or rate of change in pain (Table 3). Although race was not significantly associated with pain at the FMP or rates of change in pain, Hispanic race was associated with a greater cumulative decrease in pain over the 12year period around the FMP. Women who reported abdominal cramps at baseline had less overall bodily pain at the FMP, greater declines in pain during the late reproductive years and greater cumulative decreases in pain over the 12-year period around the FMP. Women with depression and sleep problems had greater increases in bodily pain during the late reproductive years and had greater cumulative increases over the 12-year period around the FMP (Table 3).

\section{Discussion}

To our knowledge, this study is the first to examine changes in bodily pain in relation to time to/from the FMP. We observed statistically significant differences in the rates of change in bodily pain during the transition from transmenopause to postmenopause, but the magnitude of these changes was small. The average rates of change were an increase of 0.26 per year during the 4.5 years before the FMP to 0.5 years after the FMP and a decrease of 0.23 per year during the 0.5 to 8.4 years after the FMP. These numbers correspond to a mean increase of 1.30 (out of 100) over the five-year transmenopause period and a mean decrease of 1.82 (out of 100) over the 7.9-year postmenopause period. Previous studies in individuals with osteoarthritis and rheumatoid arthritis suggest that the minimal clinically important change in SF-36 bodily pain is between 7.2 to 14.7 on a 100-point scale. ${ }^{1,}{ }^{19}$ Therefore, although the rates of change in bodily pain are statistically significant, the magnitude of these average changes are unlikely to be clinically meaningful. 
Although average changes in bodily pain were small, some women had clinically significant declines in bodily pain. For example, women who reported abdominal cramps in the reproductive years had the largest declines in overall bodily pain, with an average SF-36 score that was 6.14 points below the referent group at the time of the FMP. This observation suggests that, among these women, decreases in overall bodily pain may largely be due to resolution of dysmenorrhea, a highly prevalent condition among women of reproductive age. ${ }^{17}$ In contrast, women who reported depression and sleep problems had the largest increases in bodily pain. Their cumulative increase over 12 years was 3.39 and 2.52 points greater than the referent women, respectively. These results are consistent with a growing body of literature showing that poor mood and sleep problems may precede pain and increase the risk of chronic widespread pain conditions. ${ }^{11,36}$

The slight differences in the rates of change in overall SF-36 bodily pain during the transition between the reproductive years and menopause are consistent with longitudinal analyses from the Penn Ovarian Aging Study (POAS) ${ }^{9}$ and the Seattle Midlife Women's Health Study (SMWHS). ${ }^{25}$ In the POAS, ${ }^{9}$ the frequency of women reporting aches, joint pain and stiffness increased through the late transition stage and plateaued during postmenopause. When the analyses were restricted to moderate to severe aches, joint pain and stiffness, the prevalence of pain decreased during postmenopause, consistent with the decrease in SF-36 bodily pain and pain and, specifically, pain magnitude, observed in this study. In the SMWHS, the severity of back pain increased slightly across menopausal transition stages, whereas joint pain remained the same in analyses adjusted for age. Compared to the late reproductive stage, back pain severity was, on average, 0.09 (out of 4) points higher during the early menopausal transition stage and 0.13 points higher during the late menopausal transition state. Although overall pain severity did not decrease in postmenopause, the authors did note a decrease in the proportion of women reporting pain during early postmenopause.

The substantial changes in sex steroid hormones and gonadotropins that occur during the menopause transition have been raised as possible etiologies of alterations in the experience of pain at mid life. However, current literature is insufficient to support or refute this hypothesis. ${ }^{29,30,41}$ Existing data are conflicting, suggesting that this relationship, if it exists, is highly complex. The most comprehensive data derive from studies examining changes in pain perception across the menstrual cycle in reproductive-age women. A meta-analysis showed that pain thresholds are higher during the follicular phase, when the estrogen to progesterone ratio is high, compared to the luteal phase when this ratio decreases. ${ }^{32}$ These results seem to conflict with our findings of increases in pain from 4.5 years before the FMP to 0.5 years after the FMP, a time period when the overall estrogen to progesterone ratio typically increases. However, substantial fluctuations in hormone levels during this period often result in irregular oscillations in the estrogen:progesterone ratio within each individual woman. ${ }^{28}$ Further analyses are needed to elucidate the complexities of these relationships. SWAN is uniquely positioned to examine the relations between changes in sex steroid hormones and gonadotropins and change in pain reports, a goal that will be pursued in future work. 
To aid in the interpretation of these results, we also performed secondary analyses, examining trajectories of the components of the SF-36 bodily pain measure - specifically pain magnitude and pain interference. The trajectories for the pain magnitude component of the SF-36 bodily pain measure were similar to the trajectories for overall SF-36 bodily pain, with bodily pain and pain magnitude increasing slightly during transmenopause and decreasing during postmenopause. The trajectory for pain interference, however, differed, increasing over the overall follow-up period, particularly during late postmenopause.

Several studies have reported strong associations between pain magnitude and pain interference. ${ }^{16,40}$ However, the two measures are not synonymous, and several factors, including pain quality, fatigue and poor self-efficacy, have been identified as factors that contribute to pain interference, independent of pain magnitude. ${ }^{3,15,33}$ These factors and their impact on pain magnitude and pain interference may change across the menopause transition, ${ }^{8}$ leading to discrepancies between the trajectories of pain magnitude and pain interference. To our knowledge, no studies have specifically examined pain interference during the menopause transition. In a subset of 184 women in the Seattle Midlife Women's Health Study, Woods and Mitchell reported that menopause transition factors (e.g., menopause transition stages, estrone and FSH levels) were not associated with generalized symptom interference with work and relationships during the menopause transition and early postmenopause. $^{48}$

Our study has several strengths and limitations. Important strengths include its large sample size, diverse racial and ethnic distribution and longitudinal study design. A limitation is the absence of data on specific chronic pain conditions. Because changes in bodily pain depend on the overall balance between different pain conditions, it is important to distinguish between different types and sites of pain. ${ }^{23}$ For example, migraine headaches and temporomandibular disorders increase during the menopause transition and decrease after menopause, ${ }^{18,43,46}$ whereas osteoarthritis continues to worsen with age, beyond the menopause transition. ${ }^{27}$ Studies within specific chronic pain populations may be more appropriate to identify the underlying mechanisms resulting in changes in pain during the menopause transition.

Another limitation is the generalizability of the data describing late postmenopause changes in pain interference, since these data came from only $59.5 \%$ of the cohort. These women may be inherently different from the remainder of the cohort, particularly if these women experienced the FMP at an earlier age than the general population. However, our adjusted analyses included age at FMP as a covariate, and this variable did not predict significantly different bodily pain scores at baseline or different annualized rates of change in bodily pain score.

In conclusion, this study is important because it is the first to assess rates of change in bodily pain within intervals defined by the date of the FMP. This innovative modeling method takes advantage of prospectively collected data regarding the FMP to describe the menopause transition, rather than depending on descriptions of menstrual bleeding. Although we observed statistically significant differences in rates of change in bodily pain during transmenopause and postmenopause, the magnitude of these changes was small and unlikely 
to be clinically meaningful. Future studies using trajectory analyses that include data on pain severity of specific clinical conditions may provide insight into changes in the pathophysiology of these conditions during the reproductive aging process.

\section{Supplementary Material}

Refer to Web version on PubMed Central for supplementary material.

\section{Acknowledgements}

YL's work was supported by the NIH (R01 AR064850). DHS's work was supported by SWAN and NIH-NIAMS ARK24055989. AK's work was supported by an NIA-funded, UCLA Older Americans Independence Center (5 P30-AG028748). The Study of Women's Health Across the Nation (SWAN) has grant support from the National Institutes of Health (NIH), DHHS, through the National Institute on Aging (NIA), the National Institute of Nursing Research (NINR) and the NIH Office of Research on Women's Health (ORWH) (Grants U01NR004061; U01AG012505, U01AG012535, U01AG012531, U01AG012539, U01AG012546, U01AG012553, U01AG012554, U01AG012495). The content of this article is solely the responsibility of the authors and does not necessarily represent the official views of the NIA, NINR, ORWH or the NIH.

Clinical Centers: University of Michigan, Ann Arbor - Siobán Harlow, PI 2011 - present, MaryFran Sowers, PI 1994-2011; Massachusetts General Hospital, Boston, MA - Joel Finkelstein, PI 1999 - present; Robert Neer, PI 1994 - 1999; Rush University, Rush University Medical Center, Chicago, IL - Howard Kravitz, PI 2009 - present; Lynda Powell, PI 1994 - 2009; University of California, Davis/Kaiser - Ellen Gold, PI; University of California, Los Angeles - Gail Greendale, PI; Albert Einstein College of Medicine, Bronx, NY - Carol Derby, PI 2011 present, Rachel Wildman, PI 2010 - 2011; Nanette Santoro, PI 2004 - 2010; University of Medicine and Dentistry - New Jersey Medical School, Newark - Gerson Weiss, PI 1994 - 2004; and the University of Pittsburgh, Pittsburgh, PA - Karen Matthews, PI.

NIH Program Office: National Institute on Aging, Bethesda, MD - Winifred Rossi 2012 -present; Sherry Sherman 1994 - 2012; Marcia Ory 1994 - 2001; National Institute of Nursing Research, Bethesda, MD - Program Officers.

Central Laboratory: University of Michigan, Ann Arbor - Daniel McConnell (Central Ligand Assay Satellite Services).

Coordinating Center: University of Pittsburgh, Pittsburgh, PA - Maria Mori Brooks, PI 2012 -present; Kim SuttonTyrrell, PI 2001-2012; New England Research Institutes, Watertown, MA -Sonja McKinlay, PI 1995 - 2001.

Steering Committee: Susan Johnson, Current Chair

Chris Gallagher, Former Chair

We thank the study staff at each site and all the women who participated in SWAN.

\section{References}

1. Angst F, Aeschlimann A, Stucki G. Smallest detectable and minimal clinically important differences of rehabilitation intervention with their implications for required sample sizes using WOMAC and SF-36 quality of life measurement instruments in patients with osteoarthritis of the lower extremities. Arthritis Rheum. 2001; 45:384-391. [PubMed: 11501727]

2. Berecki-Gisolf J, Begum N, Dobson AJ. Symptoms reported by women in midlife: menopausal transition or aging? Menopause. 2009; 16:1021-1029. [PubMed: 19546824]

3. Boggero IA, Rojas-Ramirez MV, Carlson CR. All Fatigue is Not Created Equal: The Association of Fatigue and its Subtypes on Pain Interference in Orofacial Pain. Clin J Pain. 2016 Epub ahead of print.

4. Chlebowski RT, Cirillo DJ, Eaton CB, Stefanick ML, Pettinger M, Carbone LD, Johnson KC, Simon MS, Woods NF, Wactawski-Wende J. Estrogen alone and joint symptoms in the Women's Health Initiative randomized trial. Menopause. 2013; 20:600-608. [PubMed: 23511705]

5. Comstock GW, Helsing KJ. Symptoms of depression in two communities. Psychol Med. 1976; 6:551-563. [PubMed: 1005571] 
6. de Leon-Casasola OA. Opioids for chronic pain: new evidence, new strategies, safe prescribing. Am J Med. 2013; 126:S3-11. [PubMed: 23414718]

7. Dugan SA, Powell LH, Kravitz HM, Everson Rose SA, Karavolos K, Luborsky J. Musculoskeletal pain and menopausal status. Clin J Pain. 2006; 22:325-331. [PubMed: 16691084]

8. Ford K, Sowers M, Crutchfield M, Wilson A, Jannausch M. A longitudinal study of the predictors of prevalence and severity of symptoms commonly associated with menopause. Menopause. 2005; 12:308-317. [PubMed: 15879920]

9. Freeman EW, Sammel MD, Lin H, Gracia CR, Pien GW, Nelson DB, Sheng L. Symptoms associated with menopausal transition and reproductive hormones in midlife women. Obstet Gynecol. 2007; 110:230-240. [PubMed: 17666595]

10. Gao HL, Lin SQ, Wei Y, Chen Y, Wu ZL. The effect of age and menopausal status on musculoskeletal symptoms in Chinese women aged 35-64 years. Climacteric. 2013; 16:639-645. [PubMed: 23347340]

11. Generaal E, Vogelzangs N, Penninx BW, Dekker J. Insomnia, sleep duration, depressive symptoms and the onset of chronic multi-site musculoskeletal pain. Sleep. 2016 Epub ahead of print.

12. Gibson CJ, Thurston RC, El Khoudary SR, Sutton-Tyrrell K, Matthews KA. Body mass index following natural menopause and hysterectomy with and without bilateral oophorectomy. Int J Obes (Lond). 2013; 37:809-813. [PubMed: 23007036]

13. Gold EB, Crawford SL, Avis NE, Crandall CJ, Matthews KA, Waetjen LE, Lee JS, Thurston R, Vuga M, Harlow SD. Factors related to age at natural menopause: longitudinal analyses from SWAN. Am J Epidemiol. 2013; 178:70-83. [PubMed: 23788671]

14. Greendale GA, Sowers M, Han W, Huang MH, Finkelstein JS, Crandall CJ, Lee JS, Karlamangla AS. Bone mineral density loss in relation to the final menstrual period in a multiethnic cohort: results from the Study of Women's Health Across the Nation (SWAN). J Bone Miner Res. 2012; 27:111-118. [PubMed: 21976317]

15. Jensen MP, Dworkin RH, Gammaitoni AR, Olaleye DO, Oleka N, Galer BS. Do pain qualities and spatial characteristics make independent contributions to interference with physical and emotional functioning? J Pain. 2006; 7:644-653. [PubMed: 16942950]

16. Jensen MP, Hoffman AJ, Cardenas DD. Chronic pain in individuals with spinal cord injury: a survey and longitudinal study. Spinal Cord. 2005; 43:704-712. [PubMed: 15968299]

17. Ju H, Jones M, Mishra G. The prevalence and risk factors of dysmenorrhea. Epidemiol Rev. 2014; 36:104-113. [PubMed: 24284871]

18. Karli N, Baykan B, Ertas M, Zarifoglu M, Siva A, Saip S, Ozkaya G, Onal AE. Impact of sex hormonal changes on tension-type headache and migraine: a cross-sectional population-based survey in 2,600 women. J Headache Pain. 2012; 13:557-565. [PubMed: 22935969]

19. Kosinski M, Zhao SZ, Dedhiya S, Osterhaus JT, Ware JE Jr. Determining minimally important changes in generic and disease-specific health-related quality of life questionnaires in clinical trials of rheumatoid arthritis. Arthritis Rheum. 2000; 43:1478-1487. [PubMed: 10902749]

20. Lerman SF, Rudich Z, Brill S, Shalev H, Shahar G. Longitudinal associations between depression, anxiety, pain, and pain-related disability in chronic pain patients. Psychosom Med. 2015; 77:333341. [PubMed: 25849129]

21. Meana M, Cho R, DesMeules M. Chronic Pain: The Extra Burden on Canadian Women. BMC Womens Health. 2004; 4(Suppl 1):S17. [PubMed: 15345080]

22. Institute of Medicine. Relieving pain in America: a blueprint for transforming prevention, care, education and research. National Academy of Sciences; 2011.

23. Meriggiola MC, Nanni M, Bachiocco V, Vodo S, Aloisi AM. Menopause affects pain depending on pain type and characteristics. Menopause. 2012; 19:517-523. [PubMed: 22334057]

24. Mishra GD, Brown WJ, Dobson AJ. Physical and mental health: changes during menopause transition. Qual Life Res. 2003; 12:405-412. [PubMed: 12797713]

25. Mitchell ES, Woods NF. Pain symptoms during the menopausal transition and early postmenopause. Climacteric. 2010; 13:467-478. [PubMed: 20497030]

26. Moilanen J, Aalto AM, Hemminki E, Aro AR, Raitanen J, Luoto R. Prevalence of menopause symptoms and their association with lifestyle among Finnish middle-aged women. Maturitas. 2010; 67:368-374. [PubMed: 20869181] 
27. Prieto-Alhambra D, Judge A, Javaid MK, Cooper C, Diez-Perez A, Arden NK. Incidence and risk factors for clinically diagnosed knee, hip and hand osteoarthritis: influences of age, gender and osteoarthritis affecting other joints. Ann Rheum Dis. 2014; 73:1659-1664. [PubMed: 23744977]

28. Prior JC, Hitchcock CL. The endocrinology of perimenopause: need for a paradigm shift. Front Biosci (Schol Ed). 2011; 3:474-486. [PubMed: 21196391]

29. Randolph JF Jr. Sowers M, Bondarenko IV, Harlow SD, Luborsky JL, Little RJ. Change in estradiol and follicle-stimulating hormone across the early menopausal transition: effects of ethnicity and age. J Clin Endocrinol Metab. 2004; 89:1555-1561. [PubMed: 15070912]

30. Randolph JF Jr. Zheng H, Sowers MR, Crandall C, Crawford S, Gold EB, Vuga M. Change in follicle-stimulating hormone and estradiol across the menopausal transition: effect of age at the final menstrual period. J Clin Endocrinol Metab. 2011; 96:746-754. [PubMed: 21159842]

31. Reitsma M, Tranmer JE, Buchanan DM, VanDenKerkhof EG. The epidemiology of chronic pain in Canadian men and women between 1994 and 2007: longitudinal results of the National Population Health Survey. Pain Res Manag. 2012; 17:166-172. [PubMed: 22606681]

32. Riley JL 3rd, Robinson ME, Wise EA, Price DD. A meta-analytic review of pain perception across the menstrual cycle. Pain. 1999; 81:225-235. [PubMed: 10431710]

33. Ryan S, McGuire B. Psychological predictors of pain severity, pain interference, depression, and anxiety in rheumatoid arthritis patients with chronic pain. Br J Health Psychol. 2016; 21:336-350. [PubMed: 26525312]

34. Santoro N. The menopausal transition. Am J Med. 2005; 118(Suppl 12B):8-13. [PubMed: 16414322]

35. Sivertsen B, Lallukka T, Petrie KJ, Steingrimsdottir OA, Stubhaug A, Nielsen CS. Sleep and pain sensitivity in adults. Pain. 2015; 156:1433-1439. [PubMed: 25915149]

36. Smith MT, Klick B, Kozachik S, Edwards RE, Holavanahalli R, Wiechman S, Blakeney P, Lezotte D, Fauerbach JA. Sleep onset insomnia symptoms during hospitalization for major burn injury predict chronic pain. Pain. 2008; 138:497-506. [PubMed: 18362052]

37. Stening KD, Eriksson O, Henriksson KG, Brynhildsen J, Lindh-Astrand L, Berg G, Hammar M, Amandusson A, Blomqvist A. Hormonal replacement therapy does not affect self-estimated pain or experimental pain responses in post-menopausal women suffering from fibromyalgia: a doubleblind, randomized, placebo-controlled trial. Rheumatology (Oxford). 2011; 50:544-551. [PubMed: 21078629]

38. Stubbs D, Krebs E, Bair M, Damush T, Wu J, Sutherland J, Kroenke K. Sex Differences in Pain and Pain-Related Disability among Primary Care Patients with Chronic Musculoskeletal Pain. Pain Med. 2010; 11:232-239. [PubMed: 20002591]

39. Szoeke CE, Cicuttini FM, Guthrie JR, Dennerstein L. The relationship of reports of aches and joint pains to the menopausal transition: a longitudinal study. Climacteric. 2008; 11:55-62. [PubMed: 18202965]

40. Thomas JG, Pavlovic J, Lipton RB, Roth J, Rathier L, O'Leary KC, Buse DC, Evans EW, Bond DS. Ecological momentary assessment of the relationship between headache pain intensity and pain interference in women with migraine and obesity. Cephalalgia. 2016 Epub ahead of Print.

41. Vincent K, Warnaby C, Stagg CJ, Moore J, Kennedy S, Tracey I. Brain imaging reveals that engagement of descending inhibitory pain pathways in healthy women in a low endogenous estradiol state varies with testosterone. Pain. 2013; 154:515-524. [PubMed: 23318125]

42. Volkman JE, DeRycke EC, Driscoll MA, Becker WC, Brandt CA, Mattocks KM, Haskell SG, Bathulapalli H, Goulet JL, Bastian LA. Smoking Status and Pain Intensity Among OEF/OIF/OND Veterans. Pain Med. 2015; 16:1690-1696. [PubMed: 25917639]

43. Wang SJ, Fuh JL, Lu SR, Juang KD, Wang PH. Migraine prevalence during menopausal transition. Headache. 2003; 43:470-478. [PubMed: 12752752]

44. Ware JE Jr. Sherbourne CD. The MOS 36-item short-form health survey (SF-36). I. Conceptual framework and item selection. Med Care. 1992; 30:473-483. [PubMed: 1593914]

45. Ware, JE., Snow, KK., Kosinski, M., Gandeck, B. SF-36 health survey manuel and interpretation guide. The Health Institute, New England Medical Center; Boston, MA: 1993.

46. Warren MP, Fried JL. Temporomandibular disorders and hormones in women. Cells Tissues Organs. 2001; 169:187-192. [PubMed: 11455113] 
47. Weissman MM, Sholomskas D, Pottenger M, Prusoff BA, Locke BZ. Assessing depressive symptoms in five psychiatric populations: a validation study. Am J Epidemiol. 1977; 106:203-214. [PubMed: 900119]

48. Woods NF, Mitchell ES. Symptom interference with work and relationships during the menopausal transition and early postmenopause: observations from the Seattle Midlife Women's Health Study. Menopause. 2011; 18:654-661. [PubMed: 21317821]

49. Yoo JJ, Cho NH, Lim SH, Kim HA. Relationships between body mass index, fat mass, muscle mass, and musculoskeletal pain in community residents. Arthritis Rheumatol. 2014; 66:35113520. [PubMed: 25185757]

50. Zheng H, Sowers M, Karvonen-Gutierrez C, Jacobson JA, Randolph JF Jr. Harlow SD. Semiparametric Mixed Effect Model with Application to the Longitudinal Knee Osteoarthritis (OAK) Data. J Syst Cybern Inf. 2012; 10:87-93. [PubMed: 24039622] 


\section{Highlights}

- Bodily pain increases during transmenopause and decreases during postmenopause.

- In the overall population, mean changes in bodily pain were small.

- Women with a history of abdominal cramps had the largest declines in bodily pain.

- Women with depression and sleep problems had the largest increases in bodily pain. 


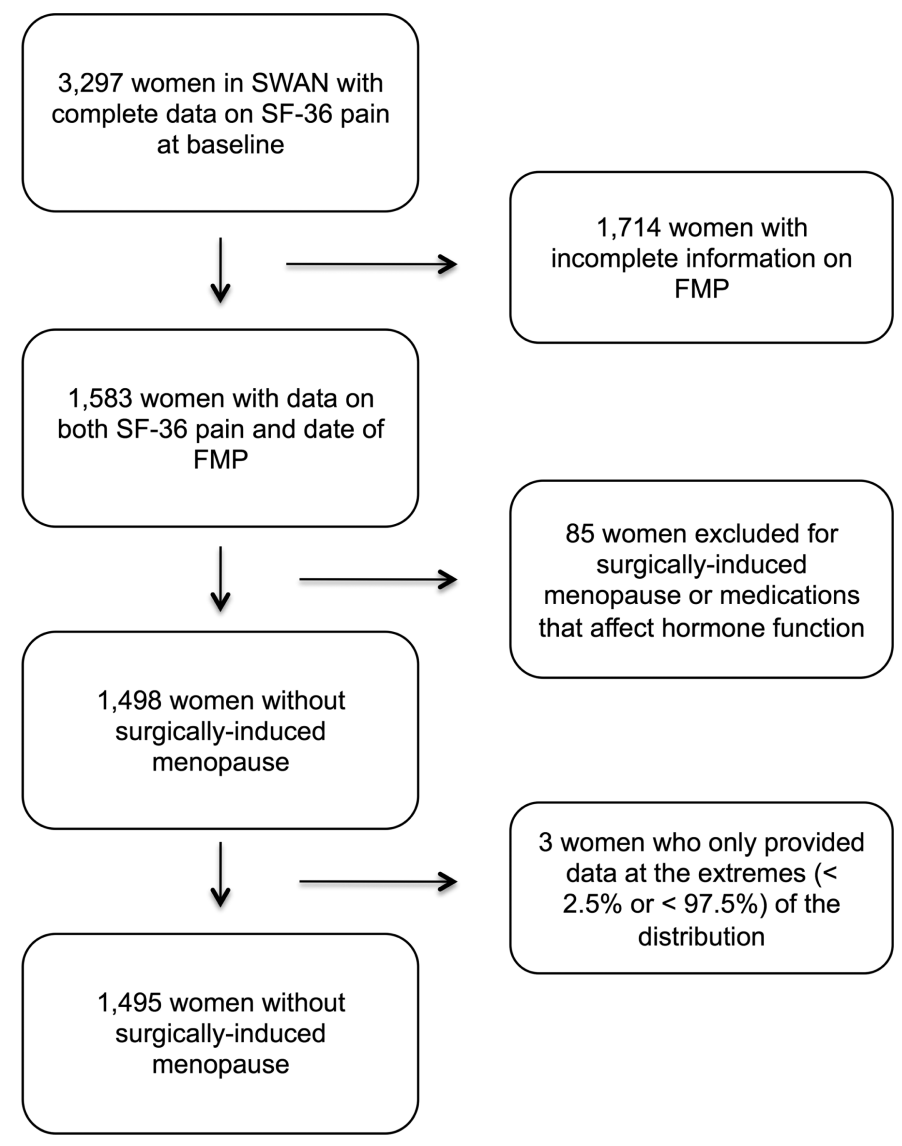

Figure 1.

Flow diagram showing the numbers of women excluded from the study and the reasons for exclusion. 


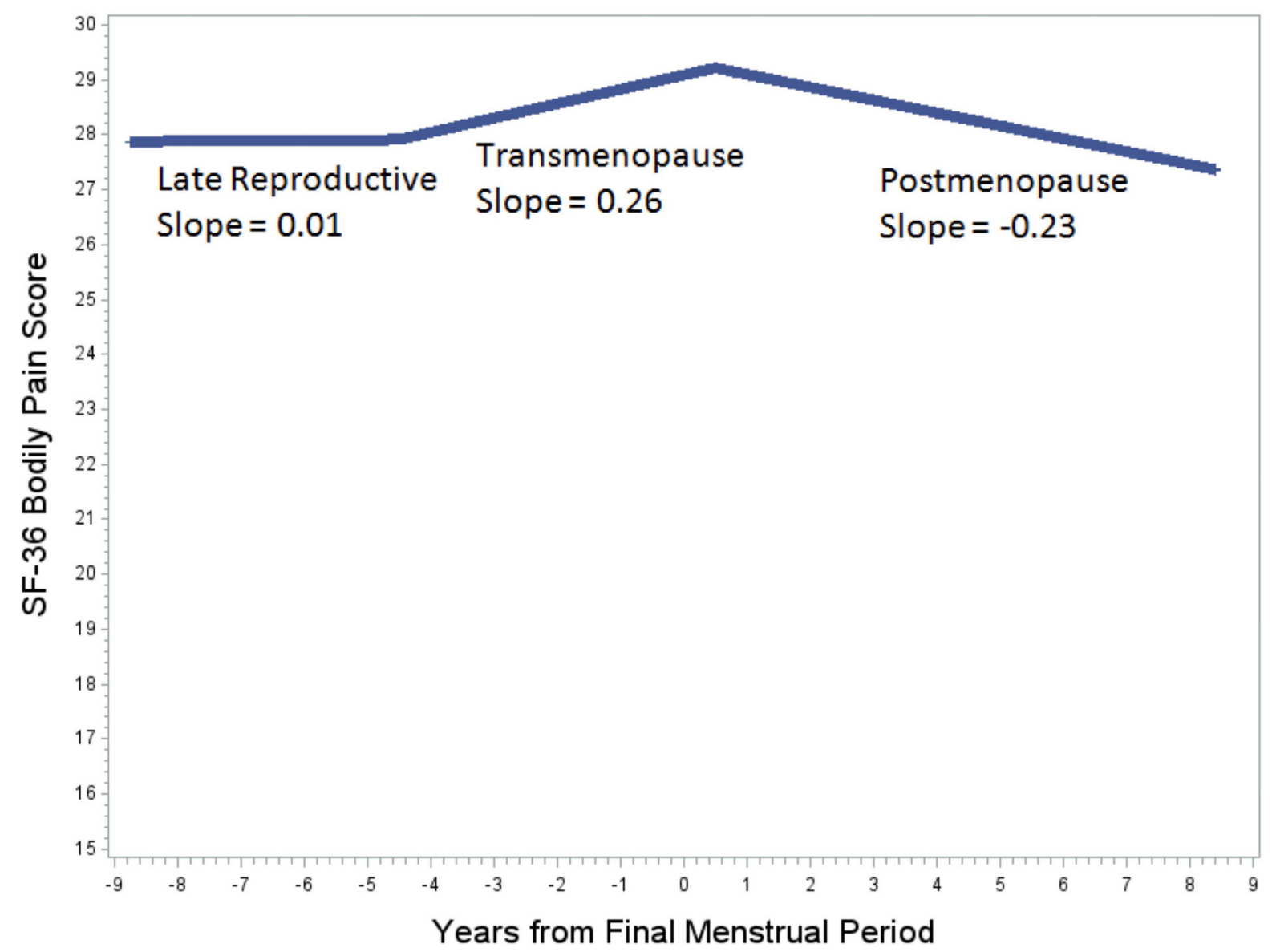

Figure 2.

The longitudinal trajectory of Short Form-36 (SF-36) pain scores in relation to the amount of time before (negative numbers) and after (positive numbers) the final menstrual period (FMP). The slopes represent the rate of change in SF-36 bodily pain during: 1) the late reproductive years: 8.8 years before the FMP to 4.5 years before the FMP, 2) transmenopause: 4.5 years before the FMP to 0.5 years after the FMP and 3 ) postmenopause: 0.55 to 8.4 years after the FMP. 
Table 1

Baseline clinical characteristics of the study population $(\mathrm{N}=1,495)$.

\begin{tabular}{|c|c|}
\hline Variable & Mean (SD)/Number (\%) \\
\hline Age (years) ${ }^{1}$ & $46.8(2.5)$ \\
\hline \multicolumn{2}{|l|}{ Race $^{1}$} \\
\hline Caucasian & $643(43.2 \%)$ \\
\hline African America & $449(30.1 \%)$ \\
\hline Japanese & $155(10.4 \%)$ \\
\hline Chinese & $145(9.7 \%)$ \\
\hline Hispanic & $98(6.6 \%)$ \\
\hline Body Mass Index $\left(\mathrm{kg} / \mathrm{m}^{2}\right)^{1}$ & $28.2(7.4)$ \\
\hline \multicolumn{2}{|l|}{ Study site ${ }^{1}$} \\
\hline Oakland, CA & $231(15.5 \%)$ \\
\hline Los Angeles, CA & $230(15.4 \%)$ \\
\hline Detroit, MI & $250(16.7 \%)$ \\
\hline Boston, MA & $236(15.8 \%)$ \\
\hline Chicago, IL & $219(14.7 \%)$ \\
\hline Pittsburgh, PA & $177(11.9 \%)$ \\
\hline Newark, NJ & $151(10.1 \%)$ \\
\hline Abdominal cramps ${ }^{1}$ & $856(57.7 \%)$ \\
\hline Current smoking ${ }^{1}$ & $236(15.9 \%)$ \\
\hline Depression & $325(21.7 \%)$ \\
\hline Sleep problems ${ }^{1}$ & $435(29.3 \%)$ \\
\hline Short Form-36 bodily pain $(0-100$ with higher number $=$ more pain $)$ & $28.7(22.1)$ \\
\hline Short Form- 36 bodily pain magnitude & $27.6(24.4)$ \\
\hline Short Form-36 bodily pain interference & $17.1(23.1)$ \\
\hline
\end{tabular}

Abbreviations: SD, standard deviation

${ }^{1}$ Due to missing data, $\mathrm{N}=1494$ for age, $\mathrm{BMI}$ and study site; $\mathrm{N}=1490$ for race; $\mathrm{N}=1486$ for sleeping problems; $\mathrm{N}=1485$ for smoking status; $\mathrm{N}=$ 1483 for abdominal cramps 


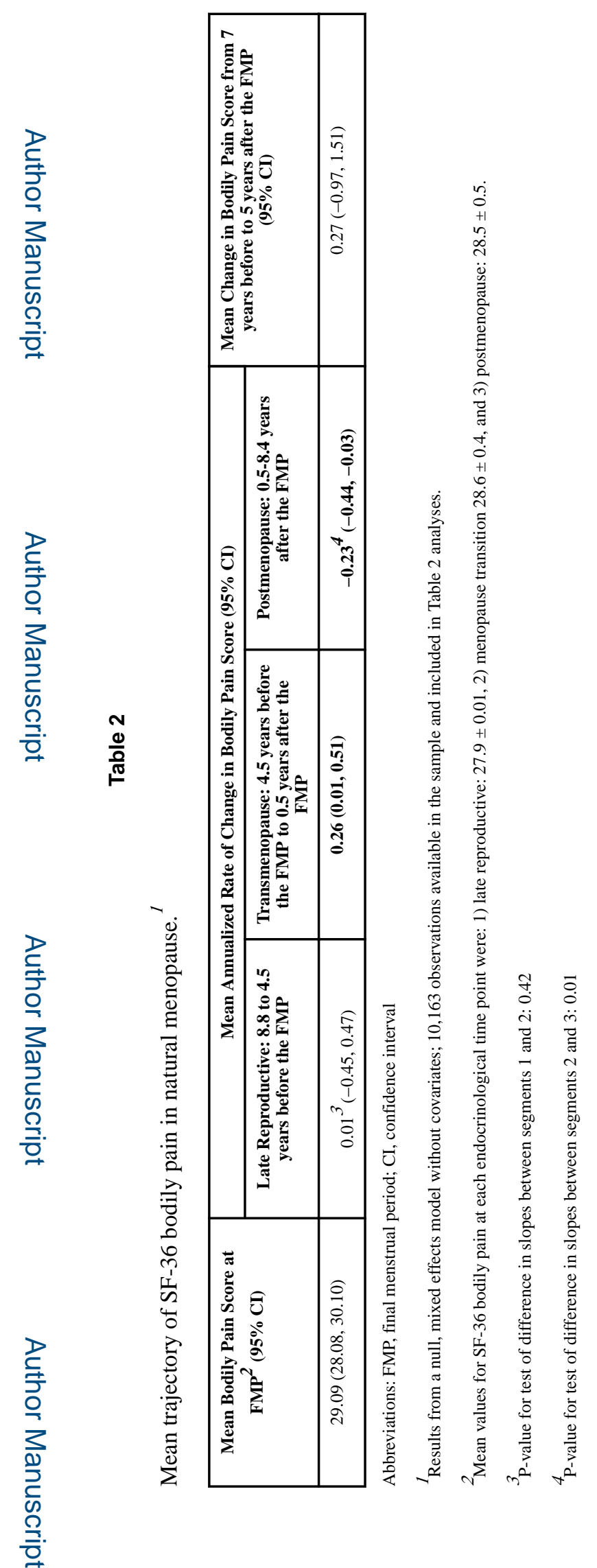

J Pain. Author manuscript; available in PMC 2018 February 01. 


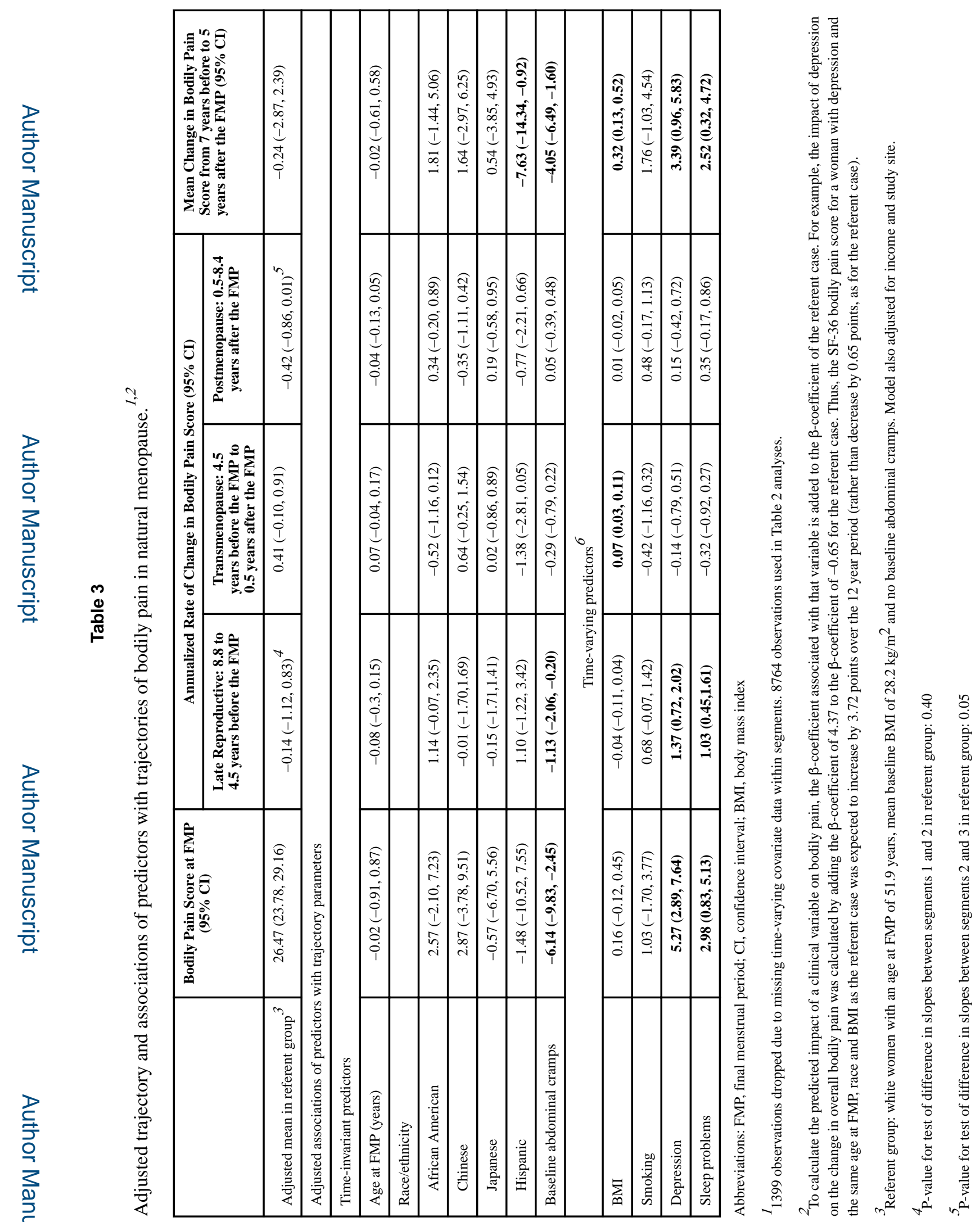

J Pain. Author manuscript; available in PMC 2018 February 01. 


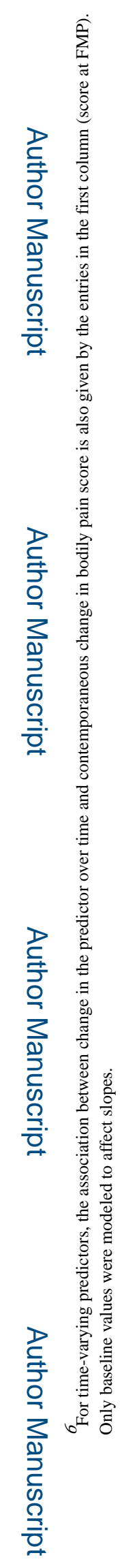

J Pain. Author manuscript; available in PMC 2018 February 01. 